\title{
Asynchronous Hybrid Duty Cycle MAC Protocol for Wireless Sensor Networks
}

\author{
Nauman Mushtaq \\ Bahria University, \\ Department of Computer Science Islamabad
}

Pakistan

\author{
Shagufta Henna \\ Bahria University \\ Department of Computer Science \\ Islamabad Pakistan
}

\begin{abstract}
Energy conversation is one of the primary objectives of duty cycle MAC protocols. Different synchronous and asynchronous duty cycle MAC protocols have been proposed in recent years. These protocols perform well under low traffic loads, but efficiency of these protocols degrade under high traffic loads. We present an asynchronous hybrid duty cycle MAC protocol called Hybrid MAC (H-MAC), which uses both sender and receiver initiated mechanisms to combat the packet delivery latency. In H-MAC each node schedules its sleep and wake up time based on cross layer routing information on the receiver initiated part and on the sender initiated part each sender chooses its wake up time based on the receiver's wake up information. We have evaluated $\mathrm{H}$ MAC in diverse network under dynamic traffic loads. Experiments reveal that, H-MAC significantly reduces packet delivery latency and energy consumption compared to RIMAC.
\end{abstract}

\section{Keywords}

Sensor networks, idle listening, duty cycling

\section{INTRODUCTION}

Wireless Sensor Networks (WSNs) have significant applications in many fields such as medical, agriculture, and military. Typically WSNs nodes have limited battery which is not rechargeable. Energy efficiency is one of the primary goal of a WSN MAC protocol. Idle listening is one of the main source of battery consumption in WSNs. In idle listening a node remains on high voltage and listens for packet transmission even when there is no communication on wireless channel resulting in wastage of energy [1]. Idle listening should be reduced in order to maximize the network lifetime.

Duty cycling is a significant and widely used mechanism to reduce idle listening in WSNs. In duty cycling each node periodically alters between active and sleep mode to conserve energy [2]. In active mode a node can transmit or receive packet data but in sleep state, it turns its radio off in order to conserves energy [3]. MAC protocols based on duty cycling for WSNs can be classified into two main categories, synchronous and asynchronous. In synchronous duty cycle MAC protocols, such as S-MAC [1], R-MAC [4], and DWMAC [5] neighboring nodes synchronize their active and sleep periods. Sender and receiver can only communicate within a common wake up time. Synchronous duty cycle approach requires synchronization among the nodes which causes extra overhead. On the other hand asynchronous duty cycle MAC protocols, such as B-MAC [3], X-MAC [6], Wise-MAC [7], RI-MAC [8], and REA-MAC [9] do not require synchronization before sending or receiving the data. Each node is independent and has its own duty cycle schedule. Asynchronous duty cycle MAC protocols are energy efficient but they cause another challenge called the time varying transmission latency. Each nodes chooses a random wake up time in an operational cycle without considering the network topology and number of hops to the destination causing significant packet delivery latency. WSNs are used for emergency applications including disaster recovery and battle field, where a faster event notification is important. Majority of duty cycle MAC protocols do not perform well under dynamic and bursty nature of traffic load.

Motivated by the problem of packet delivery latency in diverse network topologies and random traffic loads, we present a new routing enhanced hybrid sender and receiver initiated MAC protocol called H-MAC. H-MAC uses cross layer hop information to decide when a node wakes up in an operational cycle. Furthermore, H-MAC provides a hybrid receiver and sender on demand initiated mechanism to reduce the time varying latencies in packet delivery. The contribution of the work is as follows: To reduce the network latency HMAC proposes wake up mechanism based on cross layer routing information obtained from network layer. To reduce the latency and the idle listening of the sender, H-MAC proposes an on demand sender initiated mechanism on the basis of wake up time information of the receiver node. On multi-hop path H-MAC increases number of transmission in one operational cycle by using on demand sender initiated mechanism. We have evaluated the performance of H-MAC in diverse network scenarios including grid and random networks. Results reveal that H-MAC outperforms RI-MAC in terms of packet delivery ratio, energy efficiency, and packet delivery ratio.

\section{RELATED WORK}

Much synchronous and asynchronous duty cycle MAC protocols are proposed in the literature and they are energy efficient. Ye et al. [1] proposed S-MAC it was one of the original synchronous duty cycle MAC protocols. In S-MAC, neighboring nodes form clusters and they used different sleep and active schedules in different clusters to minimize energy consumption. However, in S-MAC node fairness and latency is less important. Polastre et al. [3] have proposed an asynchronous duty cycle MAC protocol for WSN called BMAC, where each node has independent duty cycle schedule. Each node wakes up periodically and checks if there is any activity on the radio. If there is activity on the link the node remains active for possible incoming packets. If a node wants to transmit the data packet, it has to transmit a preamble to notify the receiver. The preamble lasts for longer than receiver's sleeping interval to make it sure that the receiver does not miss the data packet. This long preamble increases the energy consumption by occupying the medium for a long time affecting the Packet Delivery Ration (PDR). 
In B-MAC each node hearing for the preamble transmission must stay active until the end of the preamble to find out the node interested to receive the data. Nodes which are not interested to receive the data overhear the preamble which results in significant energy consumption and packet delivery latency. X-MAC proposed by Micheal et al. [6] solves the problem of overhearing in B-MAC with a strobe preamble instead of using long preamble. Sender appends the target address in the strobe preamble. All nodes which are not interested to receive the data go to sleep immediately after receiving the short preamble. When the receiver wakes up and finds out that the data packet is meant for it, it sends an early acknowledgment, so that sender stop the preamble transmission and start the data transmission. X-MAC also reduces per hop packet delivery latency caused by long preamble in wireless sensor networks. X-MAC significantly reduces the energy consumption and per hop packet delivery latency compared to B-MAC. However short preambles in BMAC occupy wireless link for a long time which results in lower delivery of packets in one operational cycle.

RI-MAC [8] uses the idea of receiver initiated transmission [10] in WSNs. Each node wakes up periodically based on its own independent duty cycle schedule and transmits a beacon packet announcing that it is in active state. After receiving the beacon from the receiver, sender proceeds with the data transmission. After completion of first data packet receiver transmits another beacon which serves as an acknowledgment. If sender has another data packet it transmits the data if not then both the sender and receiver switch to sleep state. RIMAC minimizes the time in which sender synchronizes itself with the receiver for a communication session. It also reduces the time, in which sender occupies the wireless link for synchronization. However after receiving data sender wakes up immediately and waits until the receiver sends a beacon. This adds extra packet delivery latency and at the same time it wastes significant amount of energy due to idle listening of the sender node.

REA-MAC proposed by Wei et al. [9] uses cross layer routing information in order to decide the duty cycle schedule. REAMAC is an asynchronous duty cycle MAC protocol. REAMAC addresses the problem of idle listening in RI-MAC by using sender on demand wake up mechanism. Upon receiving a data transmission sender does not wake up immediately but wakes up based on relationship between its own wake up time and the wake up time of receiver. If sender wakes up after receiver then it has to wait for next cycle. It does not wake up immediately but wakes up in next cycle according to cross layer information. REA-MAC significantly reduces the packet deliver latency at each hop compared to RI-MAC. In the worst case if data arrives on sender, when the receiver has already completed its wake up time in one operational cycle.

In this case sender does not wake up immediately; it wakes up based on the relationship between its own wake up time and the receiver's wake up time in the next operational cycle. Although it is not consuming energy because it is in sleep mode, but it adds packet delivery latency of one extra cycle due to idle listening of the sender. Existing duty cycle protocols conserve energy at the expense of increase in packet delivery latency, which is not acceptable for time critical application. In this paper we propose a protocol called $\mathrm{H}$ MAC which conserves energy with minimum latency.

\section{H-MAC DESIGN}

\subsection{Overview}

$\mathrm{H}-\mathrm{MAC}$ is an asynchronous hybrid sender and receiver initiated MAC protocol. In receiver initiated part each node wakes up independently based on its wake up schedule in each operational cycle and transmits a beacon immediately. This beacon transmission notifies all neighbors in the transmission range, that receiver is in active state and ready to receive the data packet. After beacon transmission sender node transmits the data packet immediately. If there are multiple packets in the queue the receiver keeps listening to the channel. If there is no communication on the link after beacon transmission, node turns off its radio in order to conserve the energy.

Similar to REA-MAC, in H-MAC each node does not wake up randomly, but schedules its wake up time based on cross layer routing information. Each node wakes up according to hop distance information and forwards the packet in a sequence on the multi-hop path. Secondly, H-MAC provides an on-demand sender initiated wake up mechanism, where If a data packet arrives at the sender at the time when receiver has already completed its wake up time in this operational cycle, receiver does not wait for the next cycle and wakes up in this operation cycle on the demand of sender. In this way, H-MAC increases the number of packets transmission in one operational cycle

\subsection{Routing Enhanced wake-up mechanism in H-MAC}

R-MAC [4] uses routing Wake-up (REW) mechanism in a synchronous duty cycle MAC protocol where wake up time for each node is maintained and exchanged according to the number of hops to the sink node on a multi-hop path. REAMAC [9] uses this approach in an asynchronous MAC protocol where each node schedules its wake up time according to the hop distance to sink node in order to forward the packets like a pipeline on a multi-hop path. H-MAC uses cross layer routing information in order to forward the data packets continuously on a multi-hop path in less time where each node wakes up based on hop count to the sink node.

Typically in a WSN all nodes gather the data and report it to a sink node on a multi-hop path. The main issue is to reduce the packet delivery latency on multi-hop path. Using routing enhanced wake-up mechanism a packet can be forwarded continuously on multi-hp path in less time. After initialization, each node chooses its wake up time from 0 to $\mathrm{T}$, where $\mathrm{T}$ is time period of one operational cycle. Typically an operational cycle is the time in which a node wakes up only once, and after waking up first time a node decides its next wake up time based on the hop count to the sink node. Each node can get its routing information from its network layer header. In H-MAC Time To Live (TTL) window is used to extract hop count information. TTL count reduces each a node forwards a packet to its next hop. Nodes further away from the sink node will have greater value of TTL compared to the nodes near to sink node. A node with greater value of TTL will wake up earlier compared to a node with the smaller value of TTL. This mechanism ensures that each node decides when to wake up on multi-hop path based on the hop distance to the sink node. The nodes further away from the sink node wake up early compared to nodes nearer to sink. Next hop of each node wakes up in a sequence making it continuous forwarding on a multi-hop path. As shown in Figure 1, node 3 is on three hop distance from the sink node and therefore it has the higher value of TTL for a packet compared to node 1 and node 2 . 
According to cross layer hop information node 3 wakes up earlier compared to node 2 and node 1 . Node 2 is two hop away from the sink. Node 2 wakes up after node 3 , and receives the data packet. Node 1 will have the smaller value of TTL compared to node 2 and node 3 . Node 1 wakes up after node 2 and node 3 . Packet reaches the destination when sink node wakes.

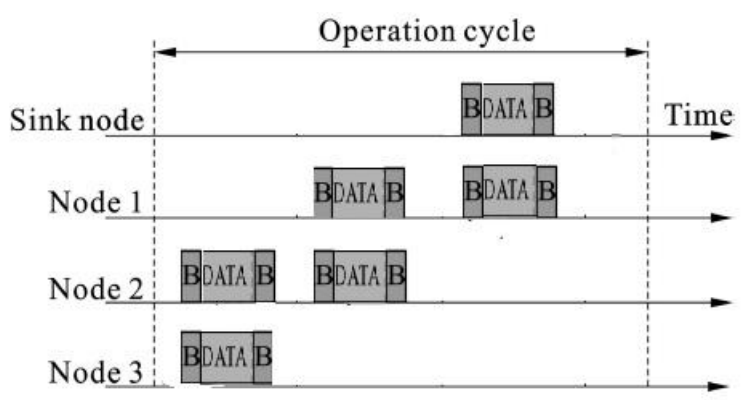

TX $\quad$ B $~ B e a c o n$

Figure 1: REW mechanism

To make it sure that a node wakes up for enough time, so that it does not go to sleep state without receiving the complete data packet. Total wake up time is calculated as follows:

Twake $=2$ Tbacon + TDATA +2 TSIFS

Where Twake is total wake up time, 2Tbacon is equal to two beacon transmission time, TDATA denoted the total time required to transmit the data packet on the wireless link and 2TSIFS is equal to two Short Interframe Spacing (SIFS), where SIFS is the time interval between a data packet and its acknowledgment. Each time a node wakes up and calculates its next wake up time according to its hop distance to the sink node, its total wake up time is computed according to Twake. REW mechanism is explained in steps given below. Ip-ttl is the TTL information from network layer header. A node with maximum value of TTL will wake up first compared to other nodes on a multi-hop path.
1: if wake up $=1$ st then wake up time $=[0-\mathrm{T}]$
2: else
3: loop:
4: if max-hop <=ip-ttl then max-hop = ip-ttl
5: if radio is sleep then wake up

\subsection{On demand Sender initiated wake up mechanism}

In this technique, we have used multiple wake-up provisioning in one operational cycle [11]. Multiple wake up provisioning allows receiving nodes to wake up multiple times in one cycle on demand of sender to avoid delay in packet delivery. When data arrives at the sender and the sender realizes that receiver has already completed its wake up time in this operational cycle. In this case instead of waiting for the next operational cycle sender transmits a wake up beacon to the receiver. The receiver wakes up on the demand of sender and receives data packet in current operational cycle. After data transmission the beacon serves as acknowledgment of the data packet completion. Both the sender and receiver go to sleep after completing the data transmission. On demand sender initiated mechanism is explained below. Time-now in the condition below is the current time when sender nodes wakes up in an operational cycle. Treceiver is the wake up time of the receiver.

If Time-now > Treceiver then

generate wake up beacon

Without using this mechanism, there can be two other ways to transmit the data packet successfully to the receiver. In the first method, sender has to wait silently for the receiver to transmit the beacon in next operational cycle as in RI-MAC [8]. In the second method sender does not wake up immediately but wakes up in the next cycle based on the relationship between its own wake up time and the wake up time of receiver as in REA-MAC [9]. Both the, RI-MAC and REA-MAC are receiver initiated MAC protocols, and they add latency due to the idle waiting of the sender. Figure 2 explains the concept of on-demand wake up mechanism. Data arrives at the sender and it realizes on the basis wake up information of neighbor, that receiver has already completed its wake up time in the current operational cycle. Sender transmits a wake up beacon to receiver and the receiver wakes up to receive the data packet. After data transmission the beacon serves as acknowledgment of the data packet completion. Both the sender and receiver go to sleep after completing the data transmission.

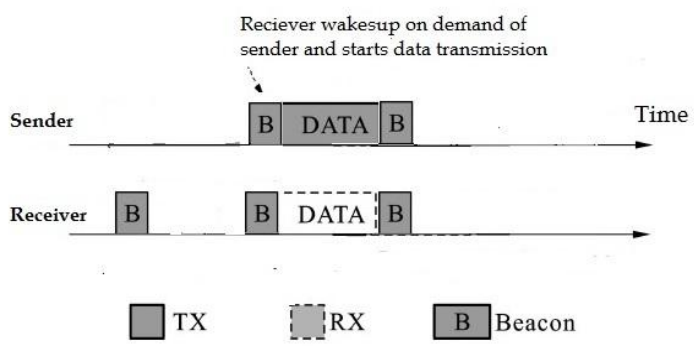

Figure 2: On demand sender initiated mechanism

\section{SIMULATION AND EVALUTION}

We evaluated both RI-MAC and H-MAC in NS-2.29. We used simulations to compare the performance of H-MAC and RI-MAC in diverse network scenarios under dynamic traffic loads. Sun et al. [8] have already compared RI-MAC with XMAC and -MAC-UPMA [6] and results show that RI-MAC outperforms them. We have used single omnidirectional antenna in our simulations. We have used TwoRayGround propagation model and simple LL link layer type. We used CBR traffic for all experiments. Other simulation parameters are given in the Table 1 .

Table 1: Simulation parameters

\begin{tabular}{|l|l|}
\hline Bandwidth & $250 \mathrm{Kbps}$ \\
\hline SIFS & $192 \mu \mathrm{s}$ \\
\hline Tx Range & $250 \mathrm{~m}$ \\
\hline Size of ACK & $5 \mathrm{~B}$ \\
\hline CCA Check Delay & $128 \mu \mathrm{s}$ \\
\hline $\begin{array}{c}\text { Carrier Sensing } \\
\text { Range }\end{array}$ & $550 \mathrm{~m}$ \\
\hline Backoff Window & 0 to 255 \\
\hline Retry Limit & 5 \\
\hline Beacon frame & $6 \mathrm{~B}$ to 9 B \\
\hline
\end{tabular}


We have used two different types of network scenarios to evaluate the performance of H-MAC including grid network, and random network. In the grid network we have varied the sensing range to evaluate the results using Random Co-related Event (RCE) model [8]. With larger sensing range more nodes report the data to sink node, traffic intensity increases with an increase in the sensing range in the grid network. For the random network we also varied the sensing range using RCE model. Sink can be anywhere and an event can occur at any $\mathrm{x}, \mathrm{y}$ location. Dynamic traffic load increase with an increase in the sensing range in the random network.

\subsection{Grid Network}

In our grid network simulation there are total 49 nodes placed in a $7 \times 7$ grid. The distance between each node is $200 \mathrm{~m}$ and the sink node is placed in the center. RCE model selects a random points at $\mathrm{x}, \mathrm{y}$ for each event. When different groups of nodes report data to sink, it forms a random correlated event. $\mathrm{R}$ is the sensing range for a random event at any $\mathrm{x}, \mathrm{y}$ location. Only nodes that are in the sensing range with radius $\mathrm{R}$ will sense the data and report it to the sink node. We have varied the sensing range $\mathrm{R}$ to simulate the results in the grid network. We varied the sensing range from $200 \mathrm{~m}$ to $1000 \mathrm{~m}$. A packet is generated after every $60 \mathrm{~s}$. Each node which senses the event sends one packet to the sink node. The length of the path from each node to sink node is 1 to 6 hops. Figure 3 exhibits the impact of different sensing ranges on average duty cycle in a grid network with RCE model. Figure 3 show that H-MAC outperforms RI-MAC in terms of duty cycle. An increase in sensing range results in an increase in traffic load because the large sensing range the more number of nodes report the data back to sink node. H-MAC experiences $0.6 \%$ duty cycle on average, on the other hand RI-MAC experiences $1 \%$ duty cycle on average. Results indicate that duty cycle of RI-MAC increase significantly beyond $600 \mathrm{~m}$ sensing range, whereas the duty cycle of H-MAC increases only $0.4 \mathrm{~s}$. Results indicate that H-MAC consumes less energy under dynamic and high traffic load compared to RI-MAC. RI-MAC has higher duty cycle due to idle listening. H-MAC has lower duty cycle due to reduced idle listening.

Figure 4 shows the impact of different sensing ranges on average latency in a grid network with RCE model. Results show that H-MAC experiences significantly less latency compared to RI-MAC with an increase in the sensing range or traffic load. In the worst case, H-MAC experiences $6 \mathrm{~s}$ less latency compared to RI-MAC for $800 \mathrm{~m}$ sensing range. $\mathrm{H}$ MAC performs better than RI-MAC due to reduced idle time, where it transmits more packets in one operation cycle.

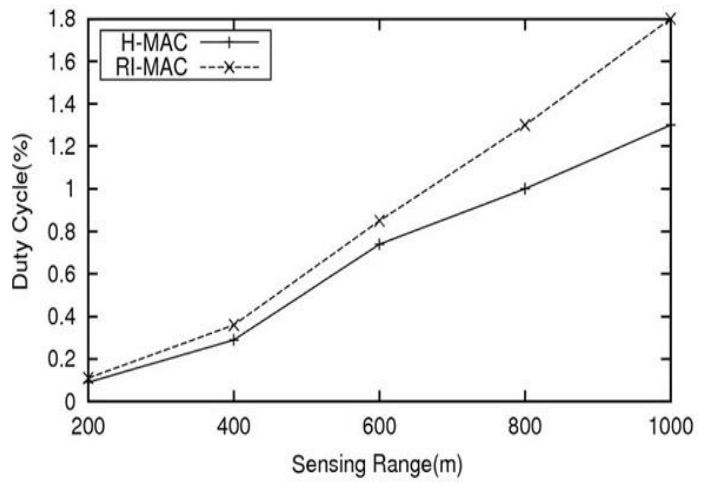

Figure 3: Duty cycle Vs sensing range

On the other hand, RI-MAC has longer latency due to sender idle time and transmits less number of packets in an operational cycle.

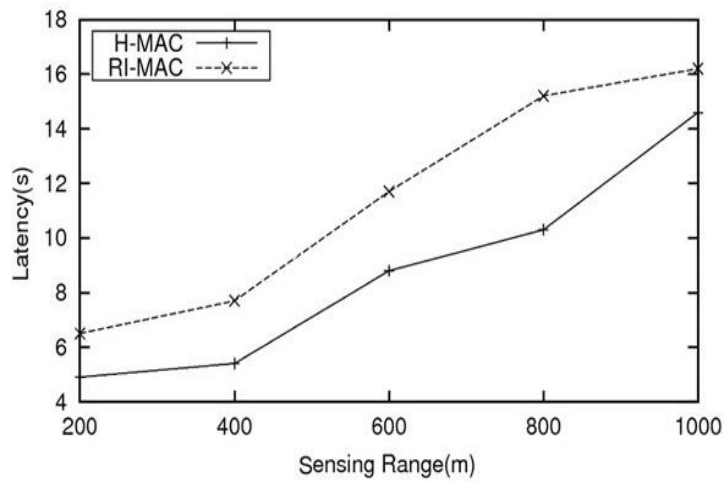

Figure 4: Latency Vs sensing range

Figure 5 shows that PDR for both RI-MAC and H-MAC always remains on $100 \%$ in grid network. There is only 1 to 6 hops distance from each node to sink; therefore there is very low probability of packet loss.

\subsection{Random Network}

We have compared RI-MAC and H-MAC in 3 different random networks. Each network has 50 nodes which are deployed in $1000 \mathrm{~m} \mathrm{X} \mathrm{100m} \mathrm{area.} \mathrm{Each} \mathrm{time} \mathrm{one} \mathrm{of} \mathrm{the} \mathrm{nodes}$ is randomly selected as a sink and all other nodes report the sensed data to sink node using. An event is generated every 30 second, where Sensing range is varied from $200 \mathrm{~m}$ to $1000 \mathrm{~m}$.

Figure 6 exhibits the impact of diverse sensing ranges on average duty cycle in random networks with RCE model in 3 runs. Results clearly show H-MAC conserves more energy compared to RI-MAC. H-MAC experiences duty cycle of $8.1 \%$ on an average, on the other hand RI-MAC experiences $10.7 \%$ duty cycle on an average. For sensing range of $1000 \mathrm{~m}$, H-MAC experiences $14 \%$ duty cycle and RI-MAC has $18.2 \%$ duty cycle. This higher duty cycle in the case of RI-MAC is due to idle listening of the nodes. H-MAC has less duty cycle because idle listening is reduced and a packet is delivered to destination by awaking the nodes on the basis of hop information.

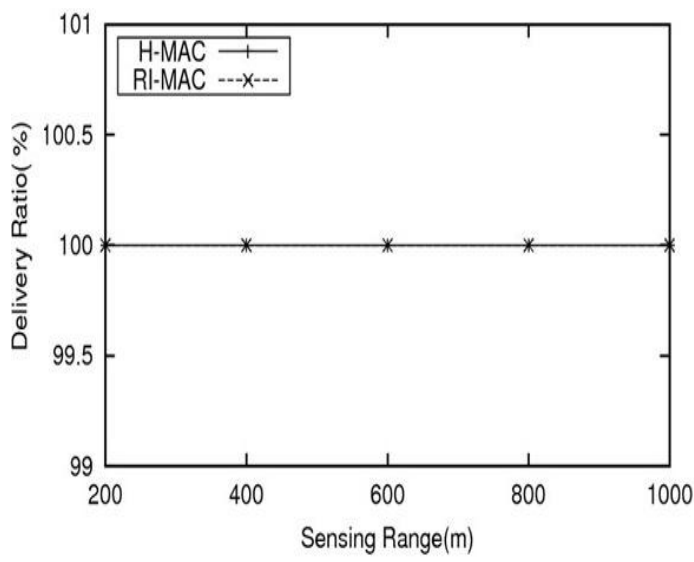

Figure 5: PDR Vs sensing range 


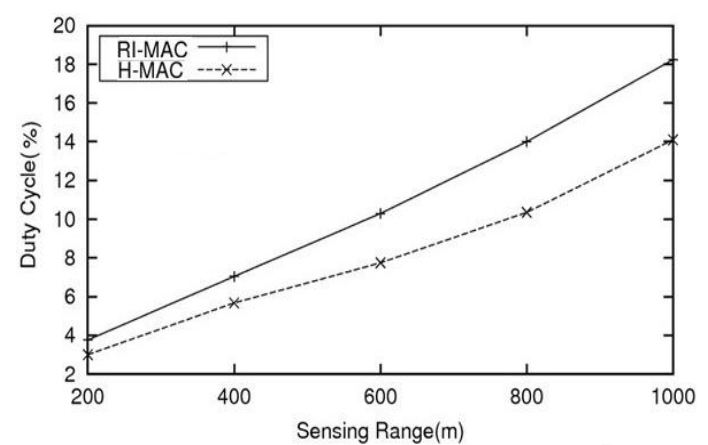

Figure 6: Duty cycle Vs sensing range

Figure 7 exhibits the impact of different sensing ranges on average latency in 3 random networks. Results show that $\mathrm{H}-$ MAC experiences lower latency compared to RI-MAC. HMAC experiences latency of 90 s on an average, on the other hand RI-MAC experiences latency of $116 \mathrm{~s}$ on an average. For sensing range of $1000 \mathrm{~m}$, RI-MAC experiences longer latency with an increase in the traffic intensity. H-MAC experiences lower latency when traffic intensity is increased for $1000 \mathrm{~m}$ sensing range. For $1000 \mathrm{~m}$ sensing range H-MAC has 170 s of latency and RI-MAC has $236 \mathrm{~s}$ of latency. RI-MAC experiences longer latency because sender nodes wait silently for the receiver to wake up in order to transmit the data packet. In H-MAC senders does not need to wait for the receiver because wake up decision is based on hop information.

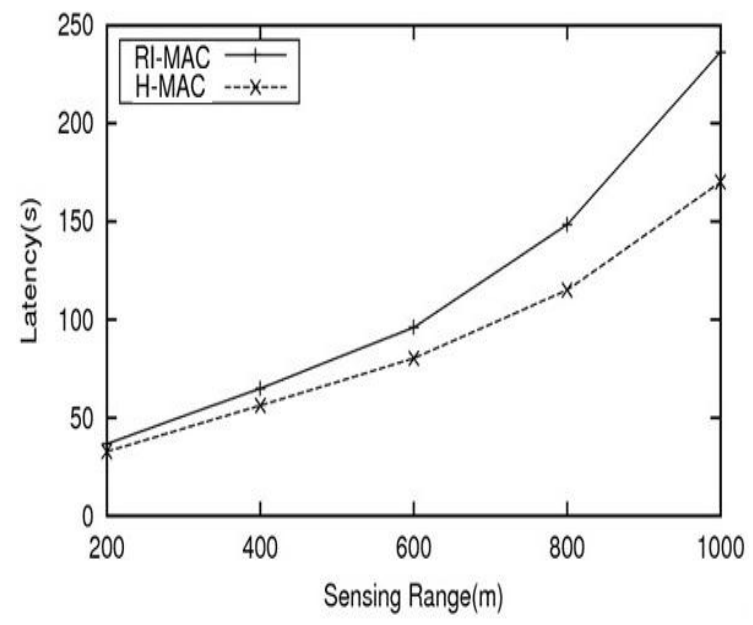

Figure 7: Latency Vs sensing range

Figure 8 shows the impact of diverse sensing ranges on PDR in 3 random networks. Results show that for different sensing ranges from $200 \mathrm{~m}$ to $800 \mathrm{~m}$ both RI-MAC and H-MAC has $100 \%$ PDR. For $1000 \mathrm{~m}$ sensing range, RI-MAC has $90 \%$ of PDR on an average. H-MAC has 97\% PDR for $1000 \mathrm{~m}$ sensing range. Results indicate that for low traffic intensity both RI-MAC and H-MAC has $100 \%$ delivery ratio, however, for increased traffic intensity, H-MAC has high PDR compared to RI-MAC. RI-MAC has low PDR due to traffic congestion in bursty and high traffic. In H-MAC, the traffic is not congested because when an event is generated, it is reported back to sink node in the form of a pipeline. the event is reported back to sink node continuously on multi-hop path.

\section{CONCLUSION}

We have presented a new asynchronous hybrid sender and receiver initiated MAC protocol, called H-MAC. H-MAC uses cross layer routing information in order to reduce the packet deliver latency. Furthermore, H-MAC uses receiver's wake up information for sender initiated data transmission in order to reduce the latency caused by idle listening of the sender. Evaluation of H-MAC through NS-2 simulation shows that H-MAC has low packet delivery latency and it conserves more energy compared to RI-MAC, furthermore H-MAC has better PDR compared to RI-MAC. Results show that H-MAC outperforms RI-MAC in diverse networks under wide range of traffic loads.

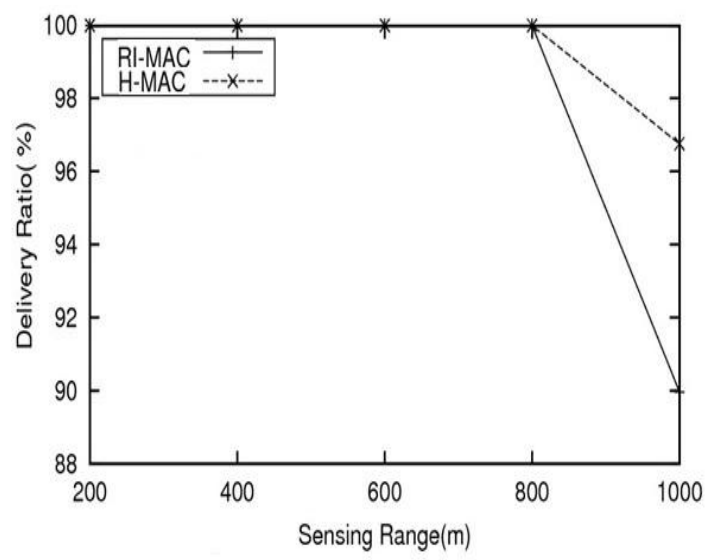

Figure 8: PDR Vs sensing range

\section{REFERENCES}

[1] Wei Ye, Estrinand, and Heidemann. An energy efficient MAC protocol for wireless sensor networks. In 21st annual joint conference of the computer and communications societies, 2002, pages 1567-1576.

[2] Yuqun zhang, Chen Hsiang Feng, Heinzelman, and Demirkol. Energy efficient duty cycle assignment for receiver based convergecast in wireless sensor networks. In Global telecommunications conference (GLOBECOM), 2010, pages 1- 5.

[3] Joseph Polastre, Jason Hill, and David Culler. Versatile low power media access for wireless sensor networks. In Proceedings of the 2nd international conference on embedded networked sensor systems, 2004, pages 95107.

[4] Kumar, Du and Johnson. RMAC: A routing enhanced duty cycle MAC protocol for wireless sensor networks. In 26th international conference on computer communications, 2007, pages 1478-1486.

[5] Yanjun Sun, Shu Du, Omer Gurewitz, and David B Johnson. DW-MAC: A low latency, energy efficient demand-wakeup MAC protocol for wireless sensor networks. In proceedings of the 9th international symposium on mobile ad hoc networking and computing, 2008, pages 53-62.

[6] Michael Buettner, Gary V. Yee, Eric Anderson, and Richard Han. XMAC: A short preambleMAC protocol for dutycycled wireless sensor. In Proceedings of the 4th international conference on embedded networked sensor systems, 2006, pages 307- 320.

[7] El-Hoiydi and Decotignie. WiseMAC: An ultra low power MAC protocol for the downlink of infrastructure wireless sensor networks. computers and communications, 2004, Volume 1:, 2004, pages 244251. 
[8] Yanjun Sun, Omer Gurewitz, and David B. Johnson. RIMAC: A receiver initiated asynchronous duty cycle MAC protocol for dynamic traffic loads in wireless sensor networks. In proceedings of the 6th conference on embedded network sensor systems, 2008, pages 1-14.

[9] Hong-wei Tang, Jian-nong Cao, Cai-xia Sun, and Kai Lu. REA-MAC: A low latency routing enhanced asynchronous duty-cycle MAC protocol for wireless sensor networks. Journal of central south university, Volume 20:, 2013, pages 678-687.
[10] Garcia Luna Aceves and Asimakis Tzamaloukas. Reversing the collision-avoidance handshake in wireless networks. In Proceedings of the 5th annual international conference on mobile computing and networking, 1999, pages $120-131$.

[11] Muhammad Mahbub Alam, Md Obaidur Rahman, Choong Seon Hong, Muhammad Mostafa Monowar, and Sungwon Lee. nW-MAC: multiple wake up provisioning in asynchronously scheduled duty cycle MAC protocol for wireless sensor networks. Annales des telecommunications, Volume 9:, 2011 